\title{
Thermal self-energies using light-front quantization
}

\author{
H. Arthur Weldon \\ Department of Physics, West Virginia University, Morgantown, West Virginia 26506-6315
}

(Dated: June 23, 2018)

\begin{abstract}
A recent paper by Alves, Das, and Perez contains a calculation of the one-loop self-energy in $\phi^{3}$ field theory at $T \neq 0$ using light-front quantization and concludes that the self-energy is different than the conventional answer and is not rotationally invariant. The changes of variable displayed below show that despite the complicated appearance of the thermal self-energy in light-front variables, it is exactly the same as the conventional result.
\end{abstract}

In Ref. 1], Alves, Das, and Perez introduce the technique of light-front quantization into thermal field theory using a heat bath at rest. As shown in [2] the appropriate quantization evolves the system in the $x^{+}=$ $\left(x^{0}+x^{3}\right) / \sqrt{2}$ coordinate while keeping constant $x^{1}, x^{2}$, and $x^{3}$. (Normal light-front quantization keeps $x^{1}, x^{2}$, and $x^{-}=\left(x^{0}-x^{3}\right) / \sqrt{2}$ constant.) The momenta conjugate to $x^{+}, x^{1}, x^{2}, x^{3}$ are $k^{0}, k^{1}, k^{2}, k^{+}$as can be seen from the identity

$$
k^{0} x^{0}-k^{3} x^{3}-\mathbf{k}_{\perp} \cdot \mathbf{x}_{\perp}=\sqrt{2} k^{0} x^{+}-\sqrt{2} k^{+} x^{3}-\mathbf{k}_{\perp} \cdot \mathbf{x}_{\perp} .
$$

In the imaginary time propagator, $x^{+}$is made negative imaginary: $-i \beta \leq \sqrt{2} x^{+} \leq 0$. In the Fourier transform of the propagator $k^{0}=i 2 \pi n T$ whereas $k^{+}$and $\mathbf{k}_{\perp}$ are real. The relation

$$
\left(k^{0}\right)^{2}-\left(k^{3}\right)^{2}-k_{\perp}^{2}=2 \sqrt{2} k^{0} k^{+}-2\left(k^{+}\right)^{2}-k_{\perp}^{2}
$$

immediately leads to the propagator

$$
G\left(k^{+}, k_{\perp}, n\right)=\frac{1}{i 4 \sqrt{2} \pi n k^{+}-2\left(k^{+}\right)^{2}-\omega_{k}^{2}},
$$

where $\omega_{k}^{2}=k_{\perp}^{2}+m^{2}$ is the transverse energy.

One of the interesting calculations performed by Alves, Das, and Perez [1] using this propagator is the one-loop self-energy for a scalar field theory with self-interaction $g \phi^{3} / 3$ !. The result of performing the summation over the loop integer $n$ is given in Eq. (40) of Ref. [1]:

$$
\Pi(p)=\frac{g^{2}}{8} \int \frac{d k^{+} d^{2} k_{\perp}}{(2 \pi)^{3}} \frac{\operatorname{coth}\left(X_{1} / 2 T\right)-\operatorname{coth}\left(X_{2} / 2 T\right)}{Y} .
$$

During the summation the external variable $p^{0}$ is an integer multiple of $2 \pi i T$, but after the summation $p^{0}$ is continued to real values. The quantities $X_{1}, X_{2}$, and $Y$ are complicated functions of the integration variables $k^{+}, \mathbf{k}_{\perp}$ and of the external variables $p^{0}, p^{+}, \mathbf{p}_{\perp}$ :

$$
\begin{aligned}
X_{1} & =\frac{\omega_{k}^{2}+2\left(k^{+}\right)^{2}}{2 \sqrt{2} k^{+}} \\
X_{2} & =\frac{\omega_{k+p}^{2}+2\left(k^{+}+p^{+}\right)^{2}}{2 \sqrt{2}\left(k^{+}+p^{+}\right)} \\
Y & =2 \sqrt{2} k^{+}\left(k^{+}+p^{+}\right)\left[-X_{1}+X_{2}-p^{0}\right] .
\end{aligned}
$$

The self-energy (1) looks quite different from the usual result and is not manifestly invariant under $O(3)$ rotations of the external momentum $\mathbf{p}$.
The following will describe a change of integration variable from $k^{+}$to a new variable $k^{3}$ that is chosen to make the self-energy a function of the two variables $p^{0}$ and $\mathbf{p}^{2}=p_{\perp}^{2}+\left(\sqrt{2} p^{+}-p^{0}\right)^{2}$. The final answer is the sum of Eqs. (2), (3), (4), and (5).

(1a) For the term $\cosh \left(X_{1} / 2 T\right) / Y$ in Eq. (1), when $k^{+}$is positive change to a new integration variable $k^{3}$ defined by

$$
k^{+}=\frac{1}{\sqrt{2}}\left[k^{3}+\sqrt{m^{2}+k_{\perp}^{2}+\left(k^{3}\right)^{2}}\right] .
$$

The range of $k^{3}$ is $-\infty \leq k^{3} \leq \infty$. The Jacobian of the transformation is $d k^{+} / d k^{3}=k^{+} / E_{k}$, where $E_{k}=$ $\sqrt{m^{2}+k^{2}}$ is the square root displayed above. Under this change,

$$
X_{1}=E_{k} ; \quad Y=k^{+}\left[\left(E_{k+p}\right)^{2}-\left(p^{0}+E_{k}\right)^{2}\right],
$$

where $E_{k+p}=\sqrt{m^{2}+(\mathbf{k}+\mathbf{p})^{2}}$. The factor $k^{+}$in the Jacobian cancels a similar factor in $Y$ and yields a contribution to the self-energy

$$
\Pi_{1 a}=\frac{g^{2}}{8} \int \frac{d^{2} k_{\perp}}{(2 \pi)^{3}} \int_{-\infty}^{\infty} \frac{d k^{3}}{E_{k}} \frac{\operatorname{coth}\left(E_{k} / 2 T\right)}{\left(E_{k+p}\right)^{2}-\left(p^{0}+E_{k}\right)^{2}} .
$$

This integrand is invariant invariant under simultaneous rotations of the vectors $\mathbf{k}$ and $\mathbf{p}$. Thus $\Pi_{1 a}$ depends only on $|\mathbf{p}|$ and $p^{0}$.

(1b) For term $\cosh \left(X_{1} / 2 T\right) / Y$ in Eq. (1), when $k^{+}$is negative make the change of variable

$$
k^{+}=\frac{1}{\sqrt{2}}\left[k^{3}-\sqrt{m^{2}+k_{\perp}^{2}+\left(k^{3}\right)^{2}}\right],
$$

where $-\infty \leq k^{3} \leq \infty$. The Jacobian of the transformation is $d k^{+} / d k^{3}=-k^{+} / E_{k}$ and

$$
X_{1}=-E_{k} ; \quad Y=k^{+}\left[\left(E_{k+p}\right)^{2}-\left(p^{0}-E_{k}\right)^{2}\right] .
$$

The corresponding self-energy contribution is

$$
\Pi_{1 b}=\frac{g^{2}}{8} \int \frac{d^{2} k_{\perp}}{(2 \pi)^{3}} \int_{-\infty}^{\infty} \frac{d k^{3}}{E_{k}} \frac{\operatorname{coth}\left(E_{k} / 2 T\right)}{\left(E_{k+p}\right)^{2}-\left(p^{0}-E_{k}\right)^{2}} .
$$

The sum of Eqs. (2) and (3) is an even function of $p^{0}$.

(2a) In the second term in Eq. (1), $\cosh \left(X_{2} / 2 T\right) / Y$, when $k^{+}+p^{+}>0$ then change to $k^{3}$ given by

$$
k^{+}=\frac{1}{\sqrt{2}}\left[k^{3}-p^{0}+\sqrt{m^{2}+\left(\mathbf{k}_{\perp}+\mathbf{p}_{\perp}\right)^{2}+\left(k^{3}+p^{3}\right)^{2}}\right],
$$


where $-\infty \leq k^{3} \leq \infty$. Using $d k^{+} / d k^{3}=\left(k^{+}+p^{+}\right) / E_{k+p}$, and

$$
X_{2}=E_{k+p} ; \quad Y=\left(k^{+}+p^{+}\right)\left[\left(p^{0}-E_{k+p}\right)^{2}-E_{k}^{2}\right] . \quad \Pi_{2 b}=\frac{g^{2}}{8} \int \frac{d^{2} k_{\perp}}{(2 \pi)^{3}} \int_{-\infty}^{\infty} \frac{d k^{3}}{E_{k+p}} \frac{\operatorname{coth}\left(E_{k+p} / 2 T\right)}{E_{k}^{2}-\left(p^{0}+E_{k+p}\right)^{2}} .
$$

This contribution is

$$
\Pi_{2 a}=\frac{g^{2}}{8} \int \frac{d^{2} k_{\perp}}{(2 \pi)^{3}} \int_{-\infty}^{\infty} \frac{d k^{3}}{E_{k+p}} \frac{\operatorname{coth}\left(E_{k+p} / 2 T\right)}{E_{k}^{2}-\left(p^{0}-E_{k+p}\right)^{2}}
$$

(2b) In the second term in Eq. (1), $\cosh \left(X_{2} / 2 T\right) / Y$, if $k^{+}+p^{+}<0$ make the change of variable

$$
k^{+}=\frac{1}{\sqrt{2}}\left[k^{3}-p^{0}-\sqrt{m^{2}+\left(\mathbf{k}_{\perp}+\mathbf{p}_{\perp}\right)^{2}+\left(k^{3}+p^{3}\right)^{2}}\right],
$$

where $-\infty \leq k^{3} \leq \infty$. Since $d k^{+} / d k^{3}=-\left(k^{+}+p^{+}\right) / E_{k+p}$, and

$$
X_{2}=-E_{k+p}, \quad Y=\left(k^{+}+p^{+}\right)\left[\left(p^{0}+E_{k+p}\right)^{2}-E_{k}^{2}\right],
$$

[1] V.S. Alves, Ashok Das, and Silvana Perez, Phys. Rev. D 66, 125008 (2002).

[2] H.A. Weldon, Phys. Rev. D 67, 0850XX (2003).
The sum of Eq. (4) and (5) is an even function of $p^{0}$.

The sum of these four contributions Eq. (2), (3), (4), and (5) is the standard answer for the self-energy [3]. Therefore the light-front formulation is a different, and in some cases a more efficient [2], way of organizing the calculation, but the results are the same.

\section{Acknowledgments}

This work was supported in part by the U.S. National Science Foundation under Grant No. PHY-0099380.
[3] A. Das, Finite Temperature Field Theory (Cambridge, University Press, Cambridge, England, 1996), page 24. 